\title{
FREQUENCY RESPONSE FUNCTION MEASUREMENT ON SIMPLIFIED DISC BRAKE MODEL
}

\author{
ÚRADNíČEK Juraj ${ }^{1}$, MUSIL Miloš² $^{2}$ BACHRATÝ Michal ${ }^{1}$ \\ ${ }^{1}$ Slovak University of Technology in Bratislava, Faculty of Mechanical Engineering, Institute of Manufacturing \\ Systems, Environmental Technology and Quality Management, Námestie slobody 17, \\ 81231 Bratislava, Slovak Republic, e - mail: juraj.uradnicek@stuba.sk \\ ${ }^{2}$ Slovak University of Technology in Bratislava, Faculty of Mechanical Engineering, Institute of Applied \\ Mechanics and Mechatronics, Námestie slobody 17, 81231 Bratislava, Slovak Republic.
}

\begin{abstract}
The paper describes role of non-proportional damping in flutter type instability, demonstrated on simplified disc brake model. The discrete two degrees of freedom system is considered to imply damping induced instability through a system eigenvalues evaluation. The Frequency Response Function (FRF) is further calculated from measurements on the physical disc brake model. From FRF, damping properties are estimated and discussed. Several different loading states of the pad versus disc are considered to show loading impact on FRF and thus damping of the system.
\end{abstract}

KEYWORDS: Brake squeal, dissipation induced instability, self-excited vibrations.

\section{Introduction}

Brake systems are important safety and performance components of vehicles as cars, trains, airplanes and industrial machines. During braking process, the components of the disc brake tend to vibrate and brake squeal can occur. It is generally accepted that brake squeal is created due to friction-induced vibration or self-excited vibration via a rotating disc [1].

There is a lot of influence which can affect the squeal. The material properties as Young's modulus, density, heat expansion as a functions of the temperature. The wear of braking parts and moisture level of environment plays also a significant role.

Unstable behavior of mechanical systems, such as disc brake, can be further caused by dissipation induced instability, which was, in the case of brake system, pointed out in the work of Hoffman and Gaul in 2003 [2]. It can be shown that this type of instability is closely related to the topology of the coefficient matrices of linearized mechanical system equations of motion. In general, damping can play both stabilizing and destabilizing role in term of spectral stability of the system, if non-conservative forces are applied to the system $[3,10]$. Purely stabilizing damping has to be of the form defined in the work [4]. System with this specific damping properties is in mechanical engineering terminology called proportionally or classically damped system and the type of damping with this stabilizing properties is known as a Rayleigh damping.

Brake system in general contains both, non-conservative forces (friction forces) [9] and presumably non-proportional damping due to high differences in material properties of the friction material and the rest of the system. Following this fact, such systems relatively easy exhibit flutter type instability. 
In recent years, a finite element (FE) approach is mostly used to predict squeal. This approach allows modeling of a mechanical system in such way, that a modal properties of a system can be analyzed and spectral stability of the system can be evaluated. Complex eigenvalue analysis is mostly used to predict unstable behavior of the system. However, no damping or only proportional damping is usually considered, especially in industrial use. This approach leads to over predictions or not accurate results compared to physical experiments [5].

In this paper, the measurement of frequency response function (FRF) to show damping properties of the simplified disc brake model is performed. Aim of this study is to highlight the importance of inclusion of a damping model into computational disc brake models to increase efficiency of squeal prediction methods.

\section{Motivation}

Dissipation induced instability has been identified several decades ago. In 1933, Smith [6] identified unstable behavior of Jeffcott rotor under specific condition when non-conservative viscous damping forces in the rotor bearings and asymmetric elastic and inertia properties of the rotor occurred. In 1952, Ziegler [7] identified decreasing limits of stability of double pendulum loaded with non-conservative follower force when minor damping was applied to the system. This problem related to brake systems was later pointed out in the work [2]. It can be shown on simplified mathematical model of brake system that stability limits can decrease with application of non-proportional damping as it was shown in the work [8]

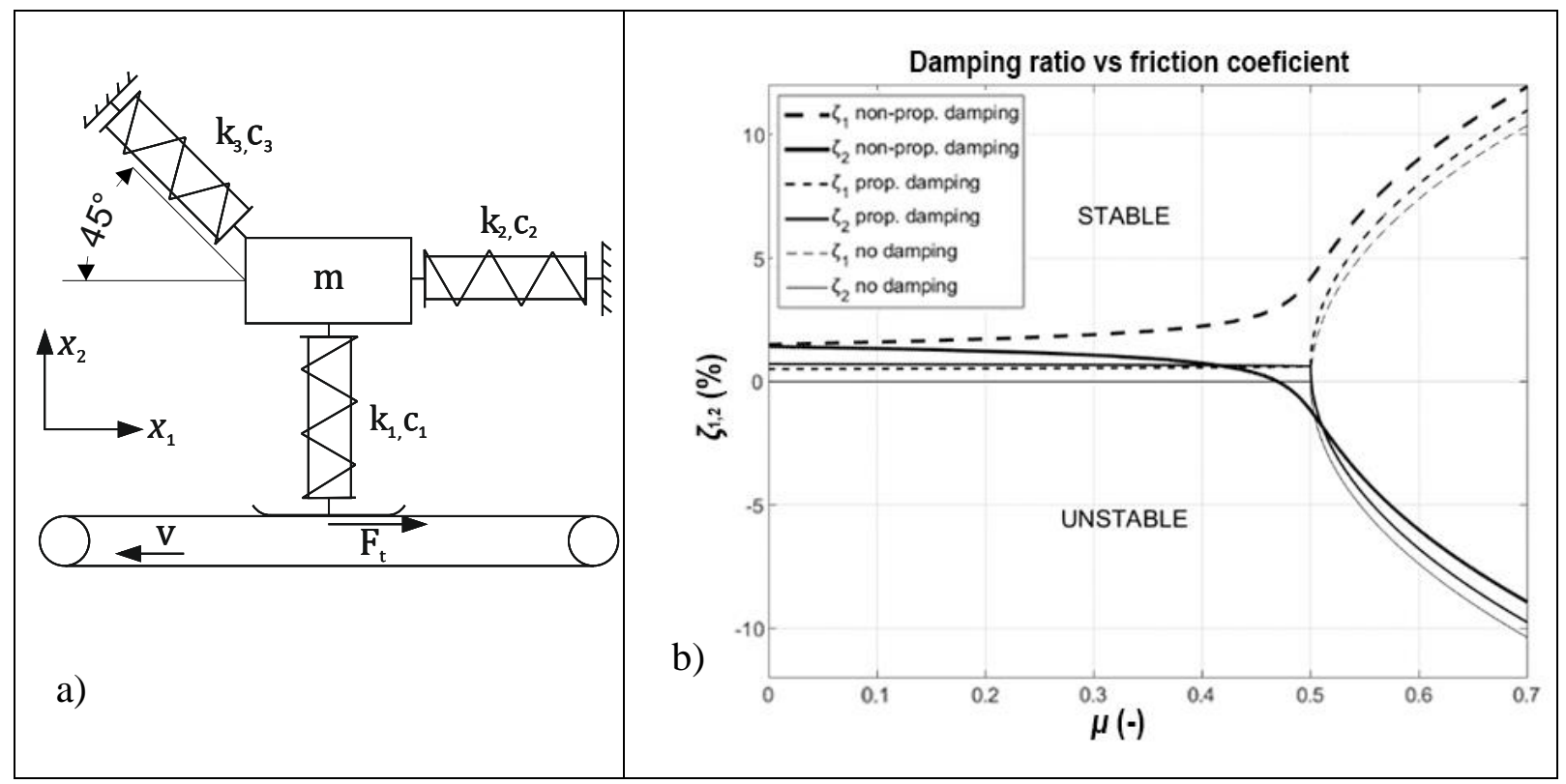

Fig. 1 a) two degrees of freedom mechanical model of disc brake, b) 1. and 2. mode damping ratios evolution with respect to friction coefficient, considering proportionally damped, nonproportionally damped and undamped system [8].

Damping ratios of the two degree of freedom discrete mechanical system at Fig. 1 a) are calculated from the system eigenvalues. The friction coefficient value represents intensity of non-conservative friction force generated due to sliding of the mass over the moving belt. The friction force, acting in the system changes its eigenvalues and thus the modal properties of the system. As it can be seen from Fig. 1 b), stability threshold value of the friction coefficient (when negative damping occures) is lower in the case of non-proportionally damped system compared to proportionally or even undamped case. In the work [4], it was 
proved, that the only stabilizing damping matrix B of any linear time invariant mass, damping, stiffness (M, B, K) system, has to be of the form

$$
\mathbf{B}=\sum_{p=0}^{m-1} c_{p} \mathbf{M}\left(\mathbf{M}^{-1} \mathbf{K}\right)^{p}, \operatorname{det} \mathbf{M} \neq 0, c_{p} \geq 0 .
$$

For the $\mathrm{m}=2$, the stabilizing damping matrix has the form of $\mathbf{B}=c_{1} \mathbf{M}+c_{2} \mathbf{K}$ which is known as a Rayleigh damping, proportional to linear combination of mass and stiffness matrix. Damping ratio of $i$-th mode of proportionally damped system can be calculated from the formula

$$
\zeta_{i}=\frac{c_{1}}{2 \omega_{i}}+\frac{c_{2} \omega_{i}}{2}
$$

where $c_{1}, c_{2}$ are constant and $\omega_{i}$ represents undamped natural frequency of $i$-th system mode.

\section{Frequency response function measurement}

The damping of the simplified disc brake model is studied in this chapter. The FRF are used to study its modal properties. The accelerometer and impact hammer was used, as it is shown at the Fig.2., to obtain time response and force input signals for FRF calculation. The FRF measurements has been done between points 1 and 2 for different loading forces F, applied on the friction material. The disc was in the static position. The aim of this study was to determine the sensitivity of structural damping to various loadings.

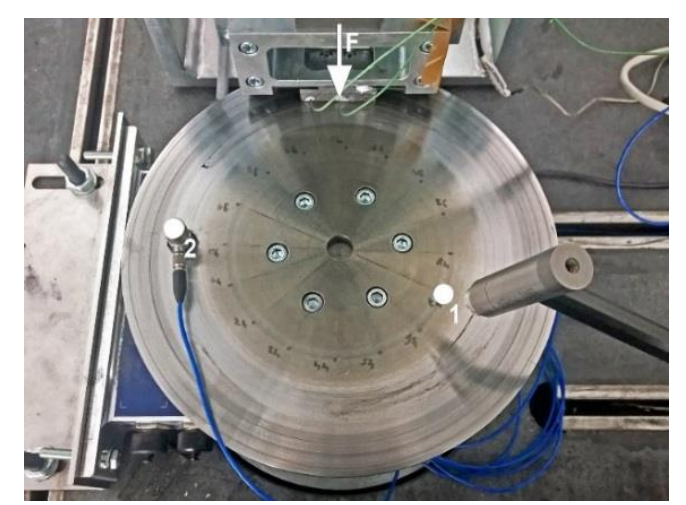

Fig. 2 FRF measurement on simplified disc brake model.

The FRF for various loadings can be seen at the Fig. 3. The regions highlighted by the vertical dashed lines are those where mode coupling and thus unstable response of the system during the braking process occurs. Most intense unstable frequencies of this particular system are in the first two regions of $3600-3800 \mathrm{~Hz}$ and $5800-6200 \mathrm{~Hz}$. 


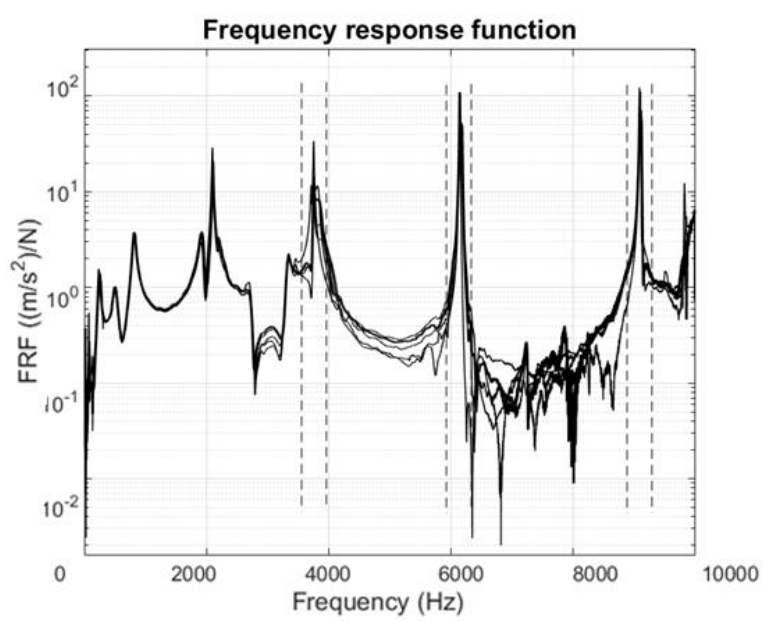

Fig. 3 Measured FRF functions with highlighted regions of squeal occurrence.
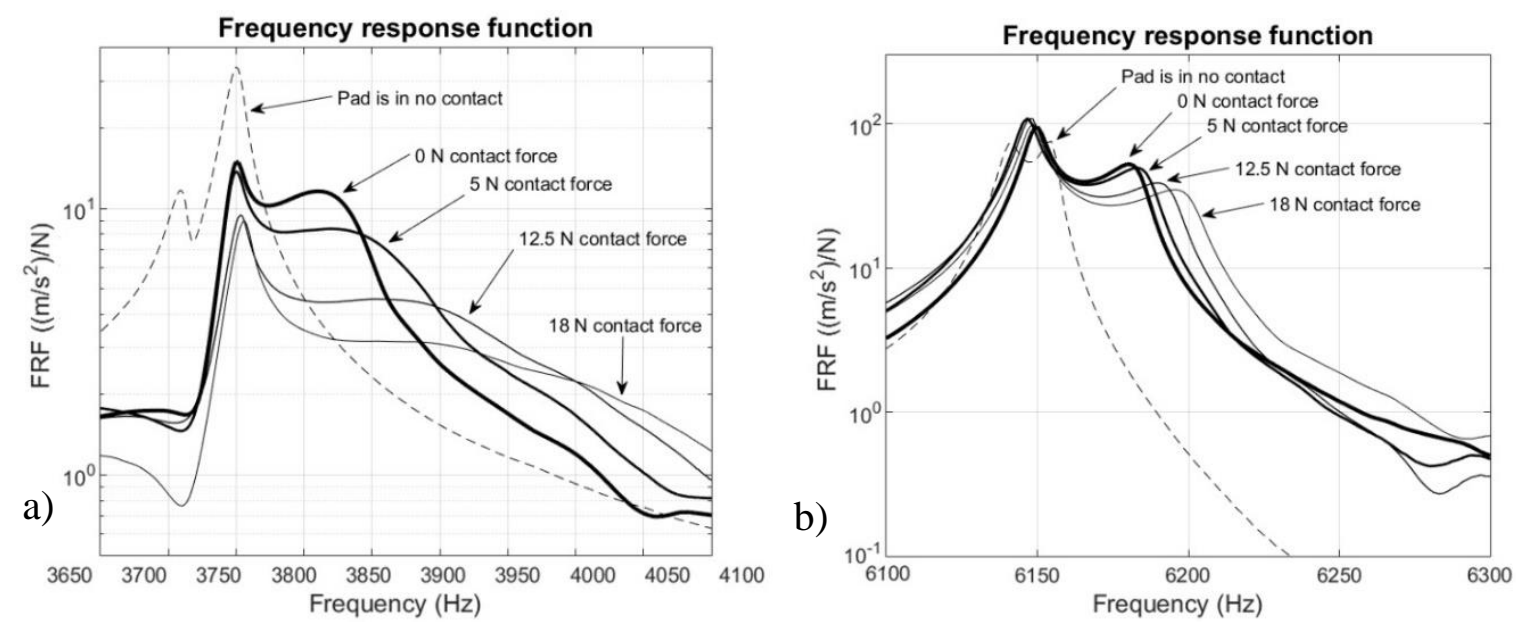

Fig. 4 Detailed comparison of FRF for different loading (F) variations in a) $3600-3800 \mathrm{~Hz}$ and b) $5800-6200 \mathrm{~Hz}$ regions.

The FRF was evaluated for five different loading cases. The dashed lines at the Fig. 4 represent the FRF on the constrained disc with no pad in the contact with it. Two close peaks with relatively same shape are obvious and represents two close modes of the system. These close modes are to be coupled if the friction force, acting on the system, reaches stability threshold value. The small frequency difference between these two modes is due to imperfect axial (mass and stiffness) symmetry of the disc and imperfect axial symmetry of the disc constrain.

According to the formula (2), the damping ratios of proportionally damped system, for any two close modes with $\omega_{i} \cong \omega_{i+1}$, should have similar values. From the almost the same shape of the lines around the peaks, it is obvious, that the unloaded disc with no friction material in the contact, acts closely to proportionally damped system. Situation differs when the pad is in the contact with the disc (Fig 4. - bold lines). The pad brings into the system additional mass, stiffness asymmetry and damping. The two close modes move further away from each other end are either shifting to the higher frequency spectrum. Furthermore, one mode from the pair exhibits bigger damping ratio which is given by its wider tip. These effects develops further with increasing brake loading applied on the pad.

From this observation it can be concluded, that although the constrained unloaded disc can be regarded as proportionally damped, the disc with pad in the contact cannot be considered 
as a proportionally damped system anymore and thus the damping induced instability can plays role in this particular system.

\section{Conclusions}

The studied disc brake system is exposed to non-conservative friction forces in combination with non-proportional damping and imperfect mass, stiffness and constrain symmetry. These combination of physical properties can lead into dynamical flutter type instability in the same way Smith [6] and Ziegler [7] pointed out on different mechanical applications several decades ago. The FRFs of simplified brake disc model has been brought out for qualitative study of system damping properties.

The study shows, that proportional damping model is proper only in the case of constrained, unloaded disc. If pad is in the contact with the disc, system exhibits strongly nonproportional damping. According to the theory and practical observations, this damping behavior allows the damping induced instability of the system by lowering the stability threshold value of non-conservative force amplitudes acting on the system.

Regarding this fact, the proper modeling of damping, in numerical models, should lead into more precise squeal prediction. Furthermore, damping brings into the disc brake model new parameter which can be optimize to help to suppress brake system instability in modal optimization process.

\section{Acknowledgement}

This work was supported by the Slovak Research and Development Agency under the contract No. APVV-15-0630.

\section{REFERENCES}

[1] A. R. Abubakar, H. Ouyang. Recent Studies Of Car Disc Brake Squeal. In. New Research on Acoustics. Nova Science Publishers 2008, 159 - 198.

[2] N. Hoffmann, L. Gaul. Effects of damping on mode-coupling instability in friction induced oscillations. Journal of Applied Mathematics and Mechanics 2003 (83), No. 3, $524-534$.

[3] M. Tommasini, O. N. Kirillov, D. Misseroni, D. Bigoni. The destabilizing effect of external damping: Singular flutter boundary for the Pflüger column with vanishing external dissipation. Journal of the Mechanics and Physics of Solids 2016 (91), 204 215.

[4] G. T. S. Done Damping configurations that have a stabilizing influence on nonconservative systems. International Journal of Solids and Structures 1973 (9), No. $2,203-215$.

[5] P. Kraus. Analysis of dynamic instability of car disc brake components considering structural-thermal effects. Dissertation thesis, Slovak University of Technology in Bratislava, 2018.

[6] D. M. Smith. The motion of a rotor carried by a flexible shaft in flexible bearings, Proc. R. Soc. Lond. A 1933 (142), No. 846, 92 - 118.

[7] H. Ziegler. Die Stabilitätskriterien der Elastomechanik. Ingenier - Archiv 1952 (20), No. $1,49-56$. 
[8] J. Úradníček, M. Musil, P. Kraus. Destabilization of the generally damped linear elastic disc brake model loaded with non-conservative friction force. In Žiaran S.: Noise and vibration in practice: peer-reviewed scientific proceedings. Spectrum STU 2018 (23), $101-106$.

[9] M. Klement, A. Gmiterko, D. Hroncová. Simulation of Worm-Like Machine. Journal of Mechanical Engineering - Strojnícky časopis 2018 (68), No. 2, 25 - 34.

[10] R. Gogola, J. Murín, J. Hrabovský. Numerical Calculation of Overhead Power Lines Dynamics. Journal of Mechanical Engineering - Strojnícky časopis 2016 (66), No 2, 13 -22 . 